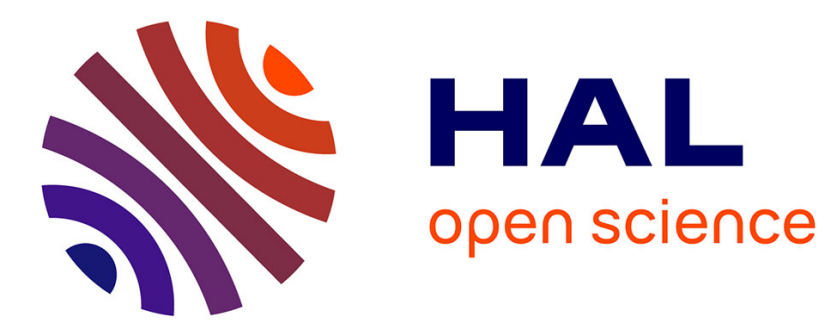

\title{
Two-channel pulse synthesis to overcome gain narrowing in femtosecond fiber amplifiers
}

Florent Guichard, Marc Hanna, Laurent Lombard, Yoann Zaouter, Clemens

Hönninger, Franck Morin, Frédéric Druon, Eric Mottay, Patrick Georges

\section{- To cite this version:}

Florent Guichard, Marc Hanna, Laurent Lombard, Yoann Zaouter, Clemens Hönninger, et al.. Twochannel pulse synthesis to overcome gain narrowing in femtosecond fiber amplifiers. Optics Letters, 2013, 38 (24), pp.5430-5433. 10.1364/OL.38.005430 . hal-00925386

\section{HAL Id: hal-00925386 \\ https://hal-iogs.archives-ouvertes.fr/hal-00925386}

Submitted on 22 Apr 2016

HAL is a multi-disciplinary open access archive for the deposit and dissemination of scientific research documents, whether they are published or not. The documents may come from teaching and research institutions in France or abroad, or from public or private research centers.
L'archive ouverte pluridisciplinaire HAL, est destinée au dépôt et à la diffusion de documents scientifiques de niveau recherche, publiés ou non, émanant des établissements d'enseignement et de recherche français ou étrangers, des laboratoires publics ou privés. 


\title{
Two-channel pulse synthesis to overcome gain narrowing in femtosecond fiber amplifiers
}

\author{
Florent Guichard, ${ }^{1,2, *}$ Marc Hanna, ${ }^{1}$ Laurent Lombard, ${ }^{3}$ Yoann Zaouter, ${ }^{2}$ Clemens Hönninger, ${ }^{2}$ \\ Franck Morin, ${ }^{2}$ Frédéric Druon, ${ }^{1}$ Eric Mottay, ${ }^{2}$ and Patrick Georges ${ }^{1}$ \\ ${ }^{1}$ Laboratoire Charles Fabry, Institut d'Optique, CNRS, Université Paris-Sud, 2 av. Augustin Fresnel, 91127 Palaiseau Cedex, France \\ ${ }^{2}$ Amplitude Systèmes, 11 avenue de Canteranne, Cité de la Photonique, 33600 Pessac, France \\ ${ }^{3}$ Office National d'Etudes et de Recherches Aérospatiales, Département d'Optique Théoriques \\ et Appliquée, Chemin de la Hunière, 91761 Palaiseau, France \\ ${ }^{*}$ Corresponding author: florent.guichard@institutoptique.fr
}

\begin{abstract}
We demonstrate spectral coherent beam combining of two femtosecond fiber chirped-pulse amplifiers seeded by a common oscillator. Using active phase stabilization based on an electro-optic phase modulator, an average power of $10 \mathrm{~W}$ before compression and a high gain factor of $30 \mathrm{~dB}$ are obtained. At this gain value, $130 \mathrm{fs}$ pulses with a spectral width of $19 \mathrm{~nm}$ can be generated, highlighting the strong potential of pulse synthesis for the reduction of the minimum duration of ultrashort pulses in fiber chirped-pulse amplifiers.
\end{abstract}

Ytterbium-doped femtosecond fiber amplifiers have numerous advantages, including high optical-to-optical efficiency, good thermal handling, and high spatial beam quality, making them suitable for the generation of high average power. However, the peak power available from these sources is limited by the ability to efficiently transfer the stored energy to a pulse as short as possible. The tight confinement of the beam inside the fiber and the large interaction length can lead to strong nonlinear effects, leading to a degraded temporal profile or optical damage. High-energy femtosecond fiber amplifiers therefore use the chirped-pulse-amplification (CPA) technique [1], along with various nonlinearity management strategies to limit the impact of a moderate amount of selfphase modulation in the system [2-4].

In these amplifiers, the achievable pulse width is limited to around $300 \mathrm{fs}$, mostly due to the gain profile of $\mathrm{Yb}$ doped fibers, which presents a strong peak at $1030 \mathrm{~nm}$ with a $\sim 40 \mathrm{~nm}$ bandwidth [5]. This spectroscopic property induces an important limitation on the achievable bandwidth during high-gain amplification, a phenomenon denoted as gain narrowing. The typical available output bandwidth is around $12 \mathrm{~nm}$ in 10-20 dB low-gain amplifiers [믈. To attenuate such problems, amplification at larger wavelengths, where the gain profile is broader, is possible [7] usually at the expense of other amplifier characteristics.

For other purposes, ultrafast physics now benefit from the development of ultrashort single-cycle sources with spectra spanning more than two octaves [8]. This was achieved by coherently adding two pulses with distinct spectra produced by two optical parametric amplifiers, where stable and long-term coherent combining was ensured through an active stabilization feedback loop. This idea was recently implemented in Yb fiber amplifiers to increase the available gain bandwidth while allowing power scaling through the coherent combination of several amplifiers. Recent results report on the development of femtosecond spectral combining and pulse synthesis with an actively stabilized feedback loop in a multichannel configuration [9] or in a multicore fiber experiment [10]. Although these results demonstrate the feasibility of spectral combining in the context of fiber amplifiers, they do not show an improvement over a single amplifier setup in terms of optical bandwidth. Quantitative results on short pulse amplification in a high gain system, comparative study of gain narrowing, and operation at high average power are still lacking.

In this Letter, we report on the development of a twochannel pulse synthesizer, which is able to amplify two distinct parts of an incoming spectrum in order to mitigate gain narrowing. For gain values larger than $30 \mathrm{~dB}$, $130 \mathrm{fs}$ pulses with a spectral width of $19 \mathrm{~nm}$ are generated in microstructured large mode area (LMA) Yb-doped fibers, at an average power level of $10 \mathrm{~W}$ and a repetition rate of $35 \mathrm{MHz}$. These results are compared to a single amplifier setup to quantify the benefits of spectral coherent combing to mitigate gain narrowing. The combining efficiency and active stabilization loop are also characterized.

The experimental setup is presented in Fig. 1 and starts with an air-cooled $\mathrm{Yb}: \mathrm{KYW}$ oscillator generating Fouriertransformed-limited $270 \mathrm{fs}$ pulses centered at $1030 \mathrm{~nm}$ with an average power of $0.5 \mathrm{~W}$ at $35 \mathrm{MHz}$ repetition rate. Output pulses are sent into a $1 \mathrm{~m}$ length standard polarization-maintaining passive fiber with $6 \mu \mathrm{m}$ mode-fielddiameter (MFD) with 50\% coupling efficiency, in order to generate a $50 \mathrm{~nm}$ spectrum through self-phase modulation. It is followed by a 400 ps grating-based $\left(1600\right.$ lines $\cdot \mathrm{mm}^{-1}$ ) stretcher designed to support $50 \mathrm{~nm}$ bandwidth pulses. This $\sim 1500$ stretching ratio is chosen to avoid nonlinear effects during amplification since spectral splitting and gain narrowing will strongly decrease the pulse duration inside the amplifiers. The coherent spectral combining setup is a Mach-Zehnder interferometer, where input/output couplers are replaced by two identical sharp-edge dichroic mirrors to ensure proper spectral separation and recombination. These 


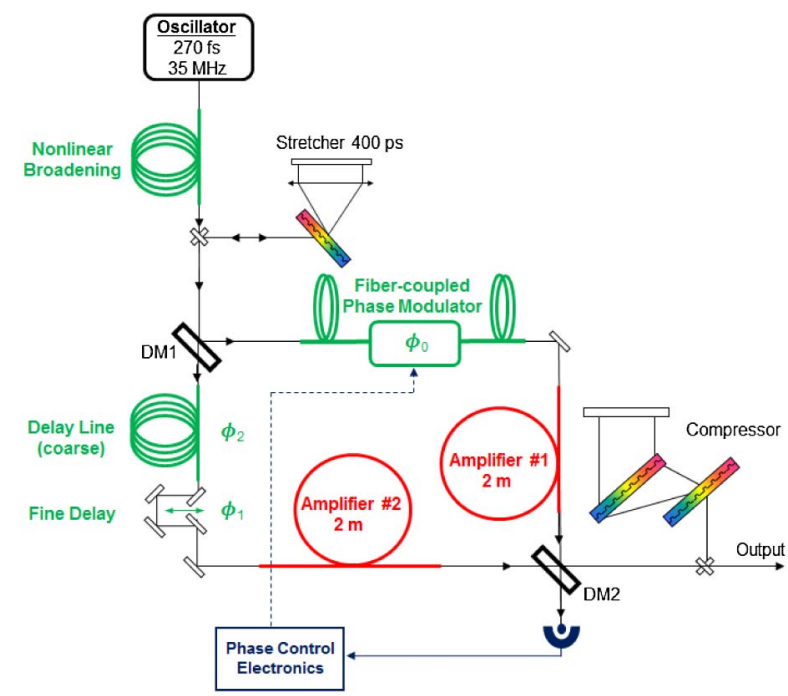

Fig. 1. Experimental setup of the pulse synthesis experiment. DM1-1: dichroïc mirror (HR: 1000-1035 nm). $\phi$ th: $n$th phase order term correction.

long-wave-pass filters are angularly adjusted to split the incident spectrum with a cutoff wavelength around $1035 \mathrm{~nm}$. A fiber-pigtailed $\mathrm{LiNbO}_{3}$ integrated phase modulator is inserted in the reflected interferometer arm (short-wavelength arm, SWA) and followed by a 2 $\mathrm{m}$-long $\mathrm{Yb}$-doped double-clad polarization-maintaining $40 / 200 \mu \mathrm{m}$ LMA fiber amplifier. The phase modulator is driven by a feedback loop to ensure active stabilization of the relative optical phase between the two interferometer arms. In the second arm (transmitted arm or longwavelength arm, LWA), 2.4 m length of passive singlemode fiber, a free-space delay line, and another $2 \mathrm{~m}$ Yb-doped double-clad 40/200 $\mu \mathrm{m}$ LMA fiber amplifier are implemented. The aim of the single-mode fiber is to compensate the second-order phase accumulated in the short-wavelength arm through propagation in the phase modulator and thus match the group-velocity dispersion of both arms while the delay line acts as a fine delay-matching element. The average power seeded to each amplifier is approximately $5 \mathrm{~mW}$. Finally, the output of each amplifier is collimated, and the optical paths from the LMA fibers end to the combining dichroic mirror are set as close as possible in order to match the spatial profiles and wavefronts of the two beams. The beam is then sent to a $60 \%$ efficiency 1600 lines $\cdot \mathrm{mm}^{-1}$ grating-based compressor.

The active stabilization loop is based on frequency tagging [11] and is similar to that described in [12]. The beam rejected by the combining dichroic mirror is detected by a photodiode, allowing us to measure the interference signal corresponding to the overlapping spectral part that is common to both arms. The tagging frequency is $250 \mathrm{kHz}$ and is added to the signal driving the phase modulator. The small induced-phase modulation is translated into an intensity modulation at the output of the interferometer and is detected by a lock-in amplifier followed by a PID controller to generate the error signal applied to the phase modulator. The overall bandwidth of this control loop is $1 \mathrm{kHz}$, which is sufficient to compensate for the environmental noise that dominates the

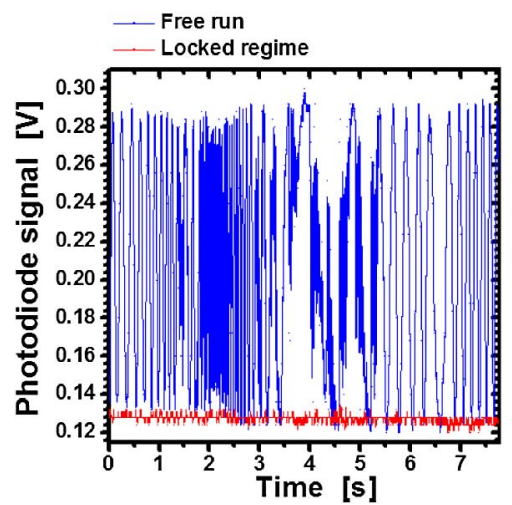

Fig. 2. Photodiode signal as a function of time in free-running and phase-locked operation.

phase fluctuations. The photodiode signal versus time is shown in Fig. 2 , both in a free-running and lockedphase regime. In the free-running regime, maximum interference contrast of $43 \%$ in the overlapping spectral part is obtained when proper group velocity and group delay matching are achieved between both interfering spectra. The system was disturbed on purpose to reveal this contrast clearly. In phase-locked operation, the signal shows residual intensity fluctuations. Taking into account the measured visibility, this corresponds to an RMS phase noise of $\lambda / 30$ over the period of $8 \mathrm{~s}$, assuming that the intensity noise is negligible. Slight readjustments of the delay line are necessary when the pump power is significantly changed due to thermo-optical and Kramers-Krönig induced refractive index change inside the amplifiers.

The setup is operated at different average-power levels in order to study the spectral coherent combining process. Figure 3(a) shows the amplifier characteristics with the combined, uncombined, and compressed average power together with the combining efficiency, defined as the ratio between the combined average power and the total output power, as a function of pump power. The combining efficiency remains constant at around $86 \%$ for all pump powers. It is slightly lower than in the case of previously demonstrated femtosecond coherent combining systems that do not perform spectral separation $[12,13]$. This is related to differences between the splitting and recombining dichroic mirrors that lead to
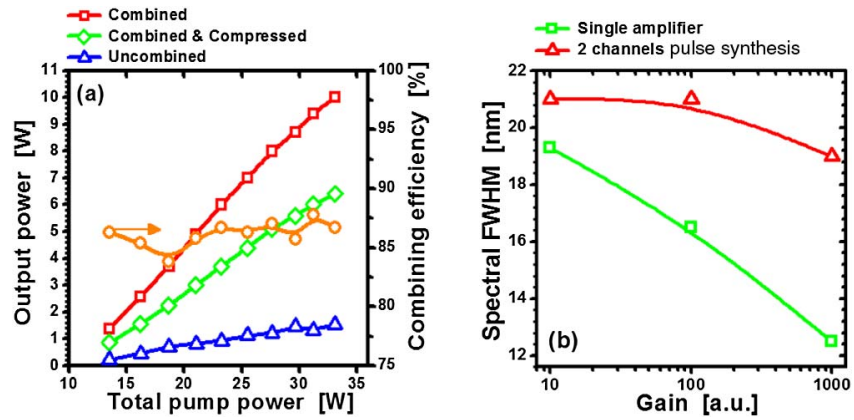

Fig. 3. (a) Combined, uncombined, and compressed average power and coherent combining efficiency as a function of pump power at $35 \mathrm{MHz}$ repetition rate. (b) Spectrum FWHM evolution for different gain levels in the two-channel (red curve) and one-channel (green curve) configurations. 

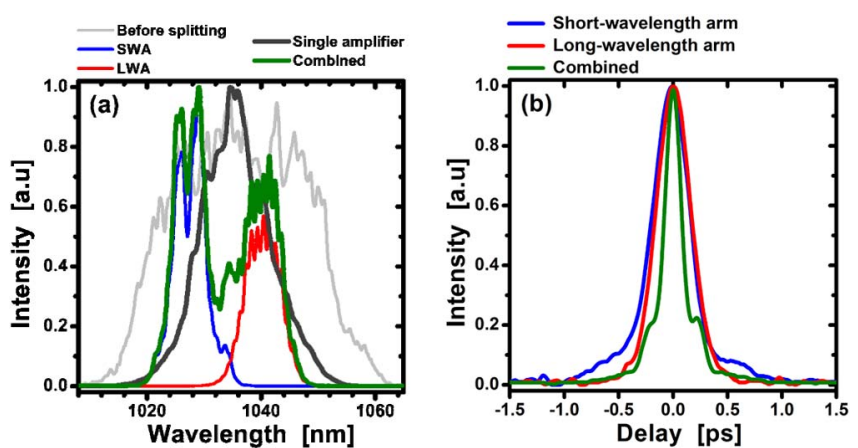

Fig. 4. (a) Experimental stretched, combined, and separately amplified arm spectra at $10 \mathrm{~W}$ combined average power together with the output spectrum in single amplifier configuration. (b) Autocorrelation trace of both arms and combined pulse.

efficiency drop and to spectral modifications during amplification that change the relative amplitude of spectral components compared to the incident spectrum, thereby affecting the combining efficiency [9]. These effects could be reduced by using steeper spectral-edge filters.

The spectral characteristics of the combined output is also studied as a function of gain value and compared to a single amplifier setup to quantify the benefit of spectral synthesis with regard to gain narrowing. The spectral FWHM of both configurations as a function of gain are plotted in Fig. 3(b). The spectral FWHM is clearly larger for the two-channel systems, highlighting the main advantage of spectral coherent combining. Gain narrowing is mitigated because narrower spectra are seeded to each amplifier, and pump levels can be adjusted to generate the largest bandwidth possible.

After careful adjustment of the pump power levels to match the average power of both arms, the obtained combined average power is $10 \mathrm{~W}$ at a $35 \mathrm{MHz}$ repetition rate. At this power level, corresponding to a gain of $30 \mathrm{~dB}$, the combined spectrum exhibits a FWHM of $19 \mathrm{~nm}$, which outperforms any reported high-gain $\mathrm{Yb}$ doped linear CPA fiber amplifier around $1030 \mathrm{~nm}$. Corresponding spectra are shown in Fig. 4(a), as well as the stretched spectrum and the amplified spectra of each channel prior to recombination. The recombined spectrum fits with the superposition of the reflected and transmitted amplified spectra; each exhibits a FWHM of approximately $7 \mathrm{~nm}$, corresponding to a stretched pulse duration of approximately $60 \mathrm{ps}$ inside each fiber amplifier. These results can be compared to the spectral width [Fig. 3(b), green-colored curve] when the full incident spectrum is seeded in a single amplifier at the same gain level. In such conditions, despite having a much broader input spectrum to seed the amplifier with, the output spectrum FWHM is only $12 \mathrm{~nm}$.

Temporal characteristics are shown in Fig. 4(b). The autocorrelation FWHM of each arm is minimum at the same compression point, indicating that the group velocity dispersion and spatial angular superposition mismatch between both beams are negligible. Indeed, minimum compressed pulse duration of both arms occurs for different compressor lengths when both beams are not perfectly superposed since different incident angles in the compressor unit change the induced spectral phase. An imperfect group-velocity dispersion matching between both arms leads to the same observation. The autocorrelation FWHM are equal to 410 fs for the SWA and 390 fs for the LWA, confirming that both arms contribute equally to the combined spectral content. The combined autocorrelation trace exhibits a FWHM of $180 \mathrm{fs}$ compared to $280 \mathrm{fs}$ when the entire incident spectrum is seeded in a single amplifier. Independent secondharmonic generation frequency-resolved optical gating (FROG) measurement reveals a combined pulse duration of 130 fs [Fig. 5(a)] and corresponding peak power of 1.9 MW. Figure 5(b) shows the retrieved autocorrelation trace, which is in good agreement with the measured autocorrelation. The FROG-retrieved spectrum is also in very good agreement with the independent spectrum measurement. The spectral phase [Fig. 5(c)] shows a low-amplitude residual structure, but overall compression quality is satisfactory as indicated by the Fourier transform-limited pulse duration of the recombined spectrum, equal to 115 fs with a corresponding peak-power of 2.4 MW. The time-bandwidth product of the synthesized pulse is 0.70 , and the temporal Strehl ratio is 0.79 .

Finally, we analyze the beam quality at the output of the compressor for both arms separately and for the combined beam (Fig. 6). $M^{2}$ measurement revealed values of, respectively, $M_{x}^{2}=1.4$ and $M_{y}^{2}=1.3$ in all cases. These values are slightly higher than those expected with such LMA amplifiers, for which $M^{2} \sim 1.1$ is a typical value, but are caused by nonoptimal end-facets surface quality.

In conclusion, we demonstrate a two-channel spectral coherent combining system, which is able to mitigate gain narrowing in a fiber amplifier. This is possible by spectrally separating a broadened incident spectrum of
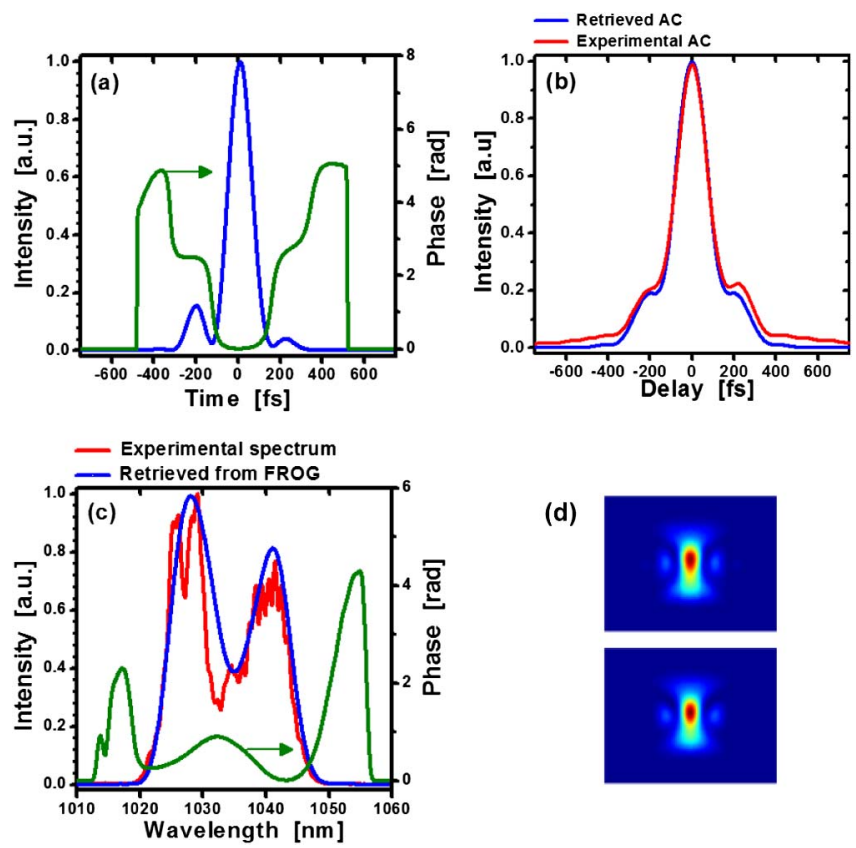

(d)

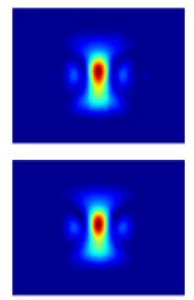

Fig. 5. (a) Retrieved temporal profile at maximum combined power. (b) Corresponding experimental and retrieved autocorrelation trace. (c) Retrieved spectrum and spectral phase together with the experimental spectrum at maximum combined power. (d) Corresponding measured (top) and retrieved (bottom) FROG traces. 


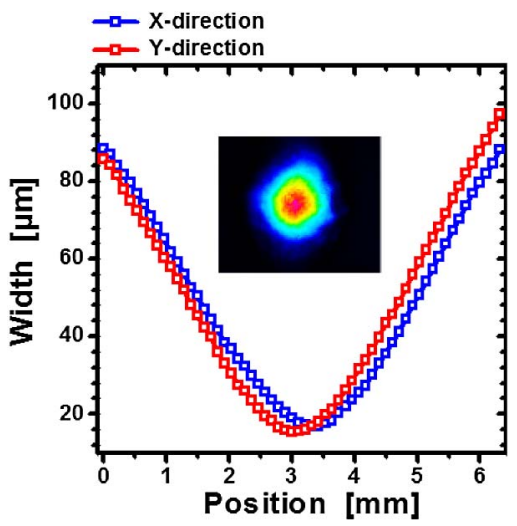

Fig. 6. (a) Output combined beam profile. Insert: Caustic of the output combined beam.

an ultrafast oscillator into two beams with distinct central wavelengths, which are amplified independently. Coherent spectral recombination is then ensured by a dichroic mirror and active phase stabilization. This scheme allows the generation of $19 \mathrm{~nm} 130 \mathrm{fs}$ pulses at $10 \mathrm{~W}$ average power and $30 \mathrm{~dB}$ gain. These results represent, to our knowledge, the first demonstration of spectral coherent combining of femtosecond fiber amplifiers at high average power and with a combined bandwidth larger than for a single channel. This experiment clearly demonstrates the advantage over a single amplifier setup in terms of output pulse duration. Shorter pulse widths can be obtained by increasing the number of spectral channels together with the implementation of a high-order dispersion management device, such as a spatial modulator-based pulse shaper. This would potentially lead to full coverage of the gain bandwidth in $\mathrm{Yb}$-doped fibers. A large number of channels also would give finer control of each spectral part, enabling spectral intensity and phase-shaping capabilities in addition to the amplification function. This technique could be implemented in large-scale, coherent combining systems to deliver ultrashort pulses at high energy and high average power.

The authors acknowledge the financial support of Agence Nationale de la Recherche through the MultiFemto project and RTRA/Labex PALM through the PulseSynth project.

\section{References}

1. D. Strickland and G. Mourou, Opt. Commun. 56, 219 (1985).

2. Y. Zaouter, J. Boullet, E. Mottay, and E. Cormier, Opt. Lett. 33, 1527 (2008).

3. S. Zhou, L. Kuznetsova, A. Chong, and F. W. Wise, Opt. Express 13, 4869 (2005).

4. D. N. Schimpf, E. Seise, T. Eidam, J. Limpert, and A. Tünnermann, Opt. Lett. 34, 3788 (2009).

5. L. Kuznetova, F. W. Wise, S. Kane, and J. Squier, Appl. Phys. B 88, 515 (2007).

6. D. Mortag, T. Theeg, K. Hausmann, L. Grüner-Nielsen, K. G. Jespersen, U. Morgner, D. Wandt, D. Kracht, and J. Neumann, Opt. Commun. 285, 706 (2012).

7. A. Ruehl, A. Marcinkevicius, M. E. Fermann, and I. Hartl, Opt. Lett. 35, 3015 (2010).

8. S.-W. Huang, G. Cirmi, J. Moses, K.-H. Hong, S. Bhardwaj, J. R. Birge, L.-J. Chen, B. J. Eggleton, G. Cerullo, and F. X. Kärtner, Nat. Photonics 5, 475 (2011).

9. W.-Z. Chang, T. Zhou, L. A. Siiman, and A. Galvanauskas, Opt. Express 21, 3897 (2013).

10. P. Rigaud, V. Kermene, G. Bouwmans, L. Bigot, A. Desfarges-Berthelemot, D. Labat, A. Le Rouge, T. Mansuryan, and A. Barthélémy, Opt. Express 21, 13555 (2013).

11. T. Shay, V. Benham, J. T. Baker, A. D. Sanchez, D. Pilkington, and C. A. Lu, IEEE J. Sel. Top. Quantum Electron. 13, 480 (2007).

12. L. Daniault, M. Hanna, L. Lombard, Y. Zaouter, E. Mottay, D. Goular, P. Bourdon, F. Druon, and P. Georges, Opt. Lett. 36, 621 (2011).

13. E. Seise, A. Klenke, J. Limpert, and A. Tünnermann, Opt. Express 18, 27827 (2010). 